\title{
Severe Persistent Hyperkalemia with Electrocardiogram Changes in a Patient with Hyperaldosteronism
}

\author{
Amr Elmoheen ${ }^{1,2}$, Larissa Michael Mishreky $^{1}$, Shadi Albeiruti ${ }^{1}$, Rabab Helmi Elanani ${ }^{1}$, Aftab Azad ${ }^{1}$ \\ 1. Emergency Department, Hamad Medical Corporation, Doha, QAT 2. Emergency Medicine, Qatar Health - College of \\ Medicine, Qatar University, Doha, QAT
}

Corresponding author: Amr Elmoheen, aelmoheen@hamad.qa

\begin{abstract}
A 62-year-old female presented to the emergency department (ED) with fatigue and generalized body weakness for the last three days. Upon arrival, initial ECG showed wide complex tachycardia with sine waves and a heart rate (HR) ranging between 100-170 bpm. She was otherwise vitally stable. The patient had a past medical history of hyperaldosteronism, type 2 diabetes mellitus (DM), chronic kidney disease (CKD) with microalbuminuria, and hypertension. She also had a history of cerebrovascular accident (CVA) and residual left-sided weakness more pronounced in the upper limb. Initial venous blood gas (VBG) analysis showed a potassium level of more than $10 \mathrm{mmol} / \mathrm{L}$, chloride $114 \mathrm{mmol} / \mathrm{L}$, bicarbonate $9 \mathrm{mmol} / \mathrm{L}$, sodium $135 \mathrm{mmol} / \mathrm{L}$, and $\mathrm{pH}$ of 7.1. Treatment for hyperkalemia was started immediately with calcium gluconate $1 \mathrm{gm}$ that effectively narrowed her QRS complex and normalized her ECG. Salbutamol nebulization, glucose/insulin infusion, and calcium polystyrene syrup were given. Later, she was started on $100 \mathrm{mg}$ sodium bicarbonate infusion, and Foley's catheter was inserted to follow urine output (UOP) strictly. However, she did not show a decrease in serum potassium levels. Then the patient underwent hemodialysis for two hours. Her first potassium reading after hemodialysis was $5.2 \mathrm{mmol} / \mathrm{L}$. The purpose of this case report is to emphasize the importance of hemodialysis in patients with persistent severe life-threatening hyperkalemia.
\end{abstract}

Review began 10/20/2020 Review ended 10/28/2020 Published 11/06/2020

(c) Copyright 2020 Elmoheen et al. This is an open access article distributed under the terms of the Creative Commons Attribution License CC-BY 4.0., which permits unrestricted use, distribution, and reproduction in any medium, provided the original author and source are credited.
Categories: Endocrinology/Diabetes/Metabolism, Emergency Medicine, Nephrology

Keywords: hyperkalemia, ecg changes, hemodialysis, hyperaldosteronism

\section{Introduction}

Hyperkalemia is caused by a range of different conditions, such as chronic kidney disease (CKD) and crush injuries, or it can be medication-induced too. Mild hyperkalemia can often be asymptomatic. However, severe hyperkalemia is a life-threatening condition that requires emergency treatment. It can lead to dangerous cardiac arrhythmias, muscle weakness, or muscle paralysis [1]. The aggressiveness of treatment greatly depends on the potassium level. Management protocol for hyperkalemia includes stopping any exogenous potassium intake, giving a calcium salt like calcium chloride or calcium gluconate, and also starting a glucose/insulin infusion. Beta-2 adrenergic drugs also aid in shifting potassium inside the cells. Sodium bicarbonate can be given to patients with metabolic acidosis, and diuretics can help in excreting potassium in the urine [2]. The potassium level is continuously monitored while administering all these drugs. Renal replacement therapy (RRT) is required for severe hyperkalemia and those not responding to drug therapy. It can clear 50-80 mmol of potassium in a four-hour dialysis session [3]. Patients with CKD are prone to hyperkalemia as kidneys are involved in potassium clearance. The presentation and outcome of hyperkalemia can be variable in such patients [4]. Also, hyperkalemia can occur in patients getting treated for hyperaldosteronism [5].

This report describes a unique presentation of life-threatening hyperkalemia in a patient with CKD and hyperaldosteronism. The patient had persistent severe hyperkalemia even after multiple treatments and only responded to hemodialysis.

\section{Case Presentation}

A 62-year-old female presented to the emergency department (ED) with the complaint of fatigue and generalized body weakness for the last three days. The patient denied any chest pain, palpitation, shortness of breath, fever, decreased urine output (UOP), recent diarrhea, dysuria, nausea, vomiting, or abdominal pain. Upon arrival, she was otherwise vitally stable with a blood pressure of $128 / 101 \mathrm{mmHg}, 37.8^{\circ} \mathrm{C}$ body temperature, and a Glasgow Coma Scale (GCS) of 15.

Initial Electrocardiogram (ECG) showed wide complex tachycardia with sine waves and a heart rate (HR) ranging between 100-170 bpm (Figure 1). 


\section{Cureus}

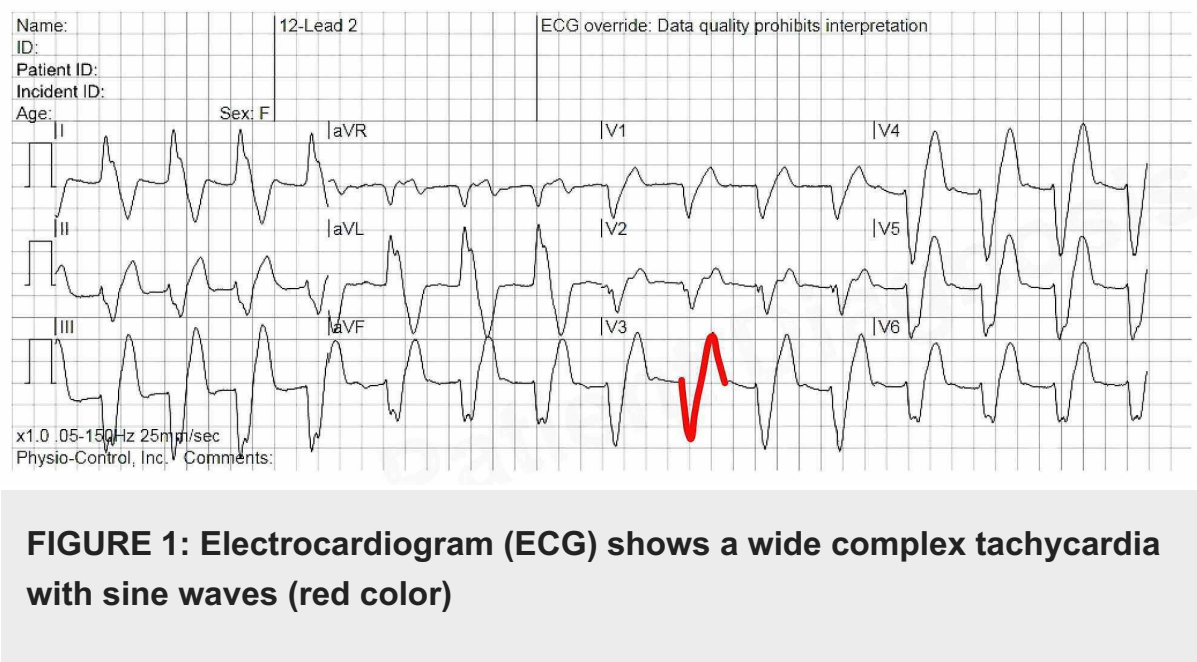

The patient had a past medical history of type 2 diabetes mellitus (T2DM), CKD with microalbuminuria, hypertension, cerebrovascular accident (CVA) with left-sided weakness more pronounced in the upper limb, and hyperaldosteronism. Her previous investigations showed a baseline creatinine of $130 \mathrm{mmol} / \mathrm{L} 1.5$ years ago. She was taking an angiotensin-converting enzyme (ACE) inhibitor - perindopril 10 milligrams (mg) daily for hypertension and metformin $500 \mathrm{mg}$ twice daily for T2DM. She was a diagnosed case of hyperaldosteronism since 2016; she was diagnosed after a period of persistent hypertension and hypokalemia. Her diagnosis was made by magnetic resonance imaging (MRI) which showed a bulky left adrenal gland with minor morphologic changes, elevated aldosterone (1,510.00 picomoles per liter (pmol/L); normal range 48-644 pmol/L) and aldosterone-renin ratio (ARR) value was higher than the cutoff. For hyperaldosteronism, she was treated medically with potassium chloride (KCL) tablets (600 mg BID) and spironolactone (500 mg once a day).

\section{Investigations}

Initial venous blood gas (VBG) analysis showed a potassium level of more than $10 \mathrm{mmol} / \mathrm{L}$, chloride 114 $\mathrm{mmol} / \mathrm{L}$, bicarbonate $9 \mathrm{mmol} / \mathrm{L}$, sodium $135 \mathrm{mmol} / \mathrm{L}$, and pH of 7.1 (Table 1). Her kidney function test showed elevated urea $(21 \mathrm{mmol} / \mathrm{L})$ and creatinine $(289 \mathrm{mmol} / \mathrm{L})$. Complete blood count $(\mathrm{CBC})$ and inflammatory markers, including C-reactive protein (CRP), lactate, and procalcitonin, were all within normal ranges.

\begin{tabular}{|c|c|c|}
\hline & Value & Normal Range \\
\hline $\mathrm{pH}$ & 7.174 & $7.320-7.420$ \\
\hline Partial Pressure of Oxygen (PO2) & $42 \mathrm{mmHg}$ & $25-40$ \\
\hline Partial Pressure of Carbon Dioxide (PCO2) & $29 \mathrm{mmHg}$ & $41-51$ \\
\hline Sodium (Na) & $137 \mathrm{mmol} / \mathrm{L}$ & $135-145$ \\
\hline Potassium (K) & $9.7 \mathrm{mmol} / \mathrm{L}$ & $3.5-5.0$ \\
\hline Chloride (Cl) & $121 \mathrm{mmol} / \mathrm{L}$ & $96-110$ \\
\hline Bicarbonate ( $\mathrm{HCO} 3$ ) & $12.0 \mathrm{mmol} / \mathrm{L}$ & $21.8-26.2$ \\
\hline
\end{tabular}

\section{TABLE 1: Venous blood gas (VBG)}

mmHg: millimeters of mercury, mmol/L: millimoles per liter

\section{Treatment}

Treatment for hyperkalemia was started immediately with intravenous administration of calcium gluconate 1 gm slowly over 10 minutes that effectively narrowed her QRS complex and normalized her ECG by restoring the normal gradient between threshold potential and resting membrane potential (Figure 2). 


\section{Cureus}

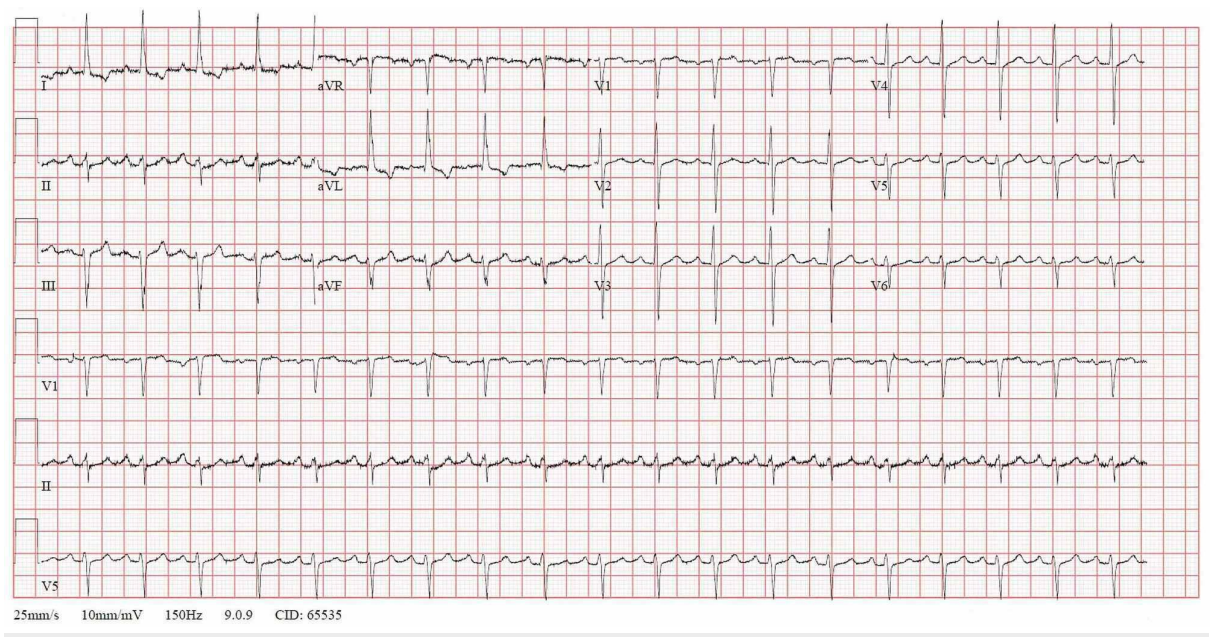

\section{FIGURE 2: Electrocardiogram (ECG) shows a narrow complex tachycardia and disappearance of the sine waves}

Salbutamol nebulization was also given twice simultaneously with the intravenous infusion of $50 \mathrm{ml}$ of dextrose $50 \%$ along with 10 units of regular insulin. Calcium polystyrene syrup (50 gm) was also given orally.

\section{Outcome}

Repeated VBG analysis showed persistently elevated potassium (more than $6.9 \mathrm{mmol} / \mathrm{L}$ ). She was given two more doses of each, glucose/insulin and salbutamol, again. VBG was repeated after these doses and showed a potassium level of above $7 \mathrm{mmol} / \mathrm{L}$.

Right after seeing no improvement in potassium level, she was started on $100 \mathrm{mg}$ sodium bicarbonate infusion, and Foley's catheter was inserted to follow UOP strictly. It drained $300 \mathrm{ml}$ of clear urine on insertion. However, she continued to have high potassium readings despite the repeated lines of treatment.

At this point, the nephrologist was involved in performing urgent hemodialysis as she was not improving. Her VBG continued to show metabolic acidosis, and the potassium level did not decrease below $6.8 \mathrm{mmol} / \mathrm{L}$. After written consent from the patient was obtained, ultrasound-guided central venous line insertion was carried out in the right femoral vein, and the patient underwent hemodialysis for two hours. Her first potassium reading after hemodialysis was $5.2 \mathrm{mmol} / \mathrm{L}$.

The patient was discharged home after changing her antihypertensive medication to oral verapamil $120 \mathrm{mg}$ daily. The patient remained asymptomatic and did not develop other attacks of hyperkalemia. She was on regular follow-up with the endocrinology and nephrology out-patient clinics.

\section{Discussion}

In our case report, severe life-threatening level of hyperkalemia causing ECG changes in this patient who did not have surgical adrenalectomy for hyperaldosteronism could be multifactorial.

The most prominent factor was the medications she was taking. The patient was taking KCL $800 \mathrm{mg}$ BID for about the last 1.5 years, and she was not following up with her primary physician during this period. Also, no follow up lab investigations like serum electrolytes were carried out during all of this period. Exogenous potassium coming from diet or supplements can cause hyperkalemia, and it is more pronounced in patients with CKD [6], which was also the case with our patient.

In addition to potassium supplements, the patient was also taking potassium-sparing diuretics spironolactone and perindopril, and both of them can also contribute to raising serum potassium level [7]. Investigations also showed an increase in serum creatinine from $133 \mathrm{mmol} / \mathrm{L}$ to $289 \mathrm{mmol} / \mathrm{L}$ during the same period that reflects the progression of CKD. However, it is not clear if it had played a major role in her presentation with hyperkalemia. CKD is associated with hyperkalemia as kidneys play a more role in potassium clearance [8]. In this particular case, the role of CKD in the patient's acute presentation is unclear as the progression of underlying CKD was not massive, and the patient was not in a state of end-stage renal disease (ESRD) given the good UOP and her estimated glomerular filtration rate (eGFR) was 15

$\mathrm{ml} / \mathrm{min} / 1.73 \mathrm{~m} 2$. Therefore, the patient's acute presentation with severe hyperkalemia seems not to be associated with her CKD. Although the patient also had metabolic acidosis at presentation, it is one of CKD's 
most common manifestations [9].

Another contributing factor to her severe hyperkalemia can be her previous stroke. She had residual leftsided weakness from the old CVA episode that could have contributed to her high potassium level. Her muscle weakness caused by stroke led to muscle wasting and immobility that can increase potassium levels [10].

The patient was treated for hyperkalemia as per protocols, and the line of treatments that involved intracellular potassium shift stabilized her initially. Still, it did not solve the main problem, i.e., the high build-up of serum potassium level throughout the last year. The patient only responded to hemodialysis after all the discussed treatment options failed to improve her hyperkalemia.

Other treatment lines that help in the excretion of potassium out of the body, such as sodium polystyrene sulfonate, were started early as well. Still, the effect is significantly delayed and questionable. Such medications treat chronic hyperkalemia in cases where the chances of complications are low and hyperkalemia is mild [11]. Therefore, it was not a very suitable treatment option for severe hyperkalemia with the risk of complications. Furosemide diuretic was also considered early in the management plan, but there was a chance of CKD progression and further elevation of creatinine [12], so it was held away.

Through this case report, we want to emphasize the importance of hemodialysis in case of severe refractory hyperkalemia with the risk of complications in patients with multiple underlying causes of hyperkalemia. If severe hyperkalemia does not respond to standard drug treatment, hemodialysis should be considered.

\section{Conclusions}

Hemodialysis helps treat hyperkalemia in cases where standard treatment does not work. In cases with multiple underlying causes of hyperkalemia, it can be difficult to determine the exact cause of acute presentation with severe hyperkalemia. In such patients who do not respond to drugs that shift potassium intracellularly or excrete it from the body, hemodialysis should be carried out to prevent life-threatening complications of severe hyperkalemia, such as cardiac arrhythmias, especially in patients with ECG changes.

\section{Additional Information}

\section{Disclosures}

Human subjects: Consent was obtained by all participants in this study. Medical Research Center, HMC, Doha-Qatar issued approval NA. Conflicts of interest: In compliance with the ICMJE uniform disclosure form, all authors declare the following: Payment/services info: All authors have declared that no financial support was received from any organization for the submitted work. Financial relationships: All authors have declared that they have no financial relationships at present or within the previous three years with any organizations that might have an interest in the submitted work. Other relationships: All authors have declared that there are no other relationships or activities that could appear to have influenced the submitted work.

\section{References}

1. Simon LV, Hashmi MF, Farrell MW: Hyperkalemia. StatPearls Publishing, Treasure Island (FL); 2020.

2. Rafique Z, Chouihed T, Mebazaa A, Peacock WF: Current treatment and unmet needs of hyperkalaemia in the emergency department. Eur Heart J. 2019, 21:A12-A19. 10.1093/eurheartj/suy029

3. Lehnhardt A, Kemper MJ: Pathogenesis, diagnosis and management of hyperkalemia. Pediatr Nephrol. 2011, 26:377-84. 10.1007/s00467-010-1699-3

4. Bianchi S, Aucella F, De Nicola L, Genovesi S, Paoletti E, Regolisti G: Management of hyperkalemia in patients with kidney disease: a position paper endorsed by the Italian Society of Nephrology. J Nephrol. 2019, 32:499-516. 10.1007/s40620-019-00617-y

5. Wada N, Shibayama Y, Umakoshi H: Hyperkalemia in both surgically and medically treated patients with primary aldosteronism. J Hum Hypertens. 2017, 31:627-32. 10.1038/jhh.2017.38

6. Batra V, Villgran V: Hyperkalemia from dietary supplements. Cureus. 2016, 8:e859. 10.7759/cureus.859

7. Chang AR, Sang Y, Leddy J: Antihypertensive medications and the prevalence of hyperkalemia in a large health system. Hypertension. 2016, 67:1181-8. 10.1161/HYPERTENSIONAHA.116.07363

8. Kim GH: Pharmacologic treatment of chronic hyperkalemia in patients with chronic kidney disease Electrolyte Blood Press. 2019, 17:1-6. 10.5049/EBP.2019.17.1.1

9. Stancu S, Mircescu G, Mocanu A, Capusa C, Stefan G: Metabolic acidosis of chronic kidney disease and cardiovascular disorders. Maedica (Bucur). 2018, 13:267-272. 10.26574/maedica.2018.13.4.267

10. Parham WA, Mehdirad AA, Biermann KM, Fredman CS: Hyperkalemia revisited. Tex Heart Inst J. 2006, 33:40-47.

11. Batterink J, Lin J, Au-Yeung SH, Cessford T: Effectiveness of sodium polystyrene sulfonate for short-term treatment of hyperkalemia. Can J Hosp Pharm. 2015, 68:296-303. 10.4212/cjhp.v68i4.1469

12. El-Refai M, Krivospitskaya O, Peterson EL, Wells K, Williams LK, Lanfear DE: Relationship of loop diuretic dosing and acute changes in renal function during hospitalization for heart failure. J Clin Exp Cardiolog. 2011, 2:1000164. 10.4172/2155-9880.1000164 